\title{
Temperature bistability in a 2D electron system on liquid helium induced by Coulomb interaction under cyclotron- resonance excitation
}

\author{
Yu.P. Monarkha \\ B. Verkin Institute for Low Temperature Physics and Engineering of the National Academy of Sciences of Ukraine \\ 47 Lenin Ave., Kharkov 61103, Ukraine \\ E-mail: monarkha@ilt.kharkov.ua
}

Received February 5, 2015, published online May 25, 2015

\begin{abstract}
The energy balance of strongly interacting surface electrons on liquid helium under cyclotron-resonance excitation is theoretically studied. The Coulomb interaction is shown to induce temperature bistability of the electron system, if the magnetic field and electron density are high enough. Surprisingly, bistability appears already for quite low average kinetic energies, when nearly all electrons occupy the ground surface subband. The electron temperature $T_{e}$, as the function of the magnetic field $B$, exhibits hysteresis and bistability jumps in a certain range of the microwave power. Above the threshold microwave field, the line shape $T_{e}(B)$ is shown to be sensitive to details of the ripplon dispersion at large wave numbers.
\end{abstract}

PACS: 73.40.-c Electronic transport in interface structures;

73.20.-r Electron states at surfaces and interfaces;

73.25.+i Surface conductivity and carrier phenomena;

78.70.Gq Microwave and radio-frequency interactions.

Keywords: cyclotron resonance, temperature bistability, surface electrons, liquid helium.

\section{Introduction}

Cyclotron resonance (CR) has proved to be a valuable research tool for studying properties of a $2 \mathrm{D}$ electron system formed on the free surface of liquid helium. In early studies [1], the 2D nature of electron states on liquid helium was demonstrated by tilting the magnetic field, and observing the shift of the resonance frequency caused by the change of the $B_{z}$ component. A broad experimental investigation of the CR line shape [2,3] provided important information about the effective mass of surface electrons (SEs) and their coupling with capillary wave quanta (ripplons). At a high excitation power, a fraction of 3D electrons evaporated from the ground surface subband is formed. The semi-classical theory [4] of this effect is in a qualitative agreement with the experiment.

SEs on liquid helium represent a highly correlated 2D electron system: the average interaction potential energy $U_{C}$ of an electron is much larger than the average kinetic energy: $U_{C}>>T$. Therefore, full understanding and theoretical description of experimentally observed linewidth data [2,3] required an analysis of quantum transport phenomena for strongly interacting electrons. In the absence of Coulomb interaction, electron scattering caused by ripplons and vapor atoms is quasi-elastic. In the selfconsistent Born approximation (SCBA), the rate of such scattering processes is enhanced [5] by the factor $\hbar \omega_{C} / \Gamma_{n}$ (here $\omega_{c}=e B / m c$ is the cyclotron frequency, and $\Gamma_{n}$ is the collision broadening of Landau levels), as compared to the semi-classical result, because electron states are squeezed into extremely narrow Landau levels: $\Gamma_{n}<<T<\hbar \omega_{c}$. Qualitatively, this factor can be considered also as a number of multiple scattering events $\omega_{C} \tau_{B}$ within the lifetime $\tau_{B} \sim \hbar / \Gamma_{n}$. The many-electron treatment of the quantum magnetotransport of SEs is based on the idea [6,7] that under a high magnetic field an internal electric filed, acting on an electron displaced from an equilibrium position due to fluctuations, can be considered as a quasi-uniform field. This fluctuational electric field $E_{f}$ drives the cyclotron orbit center away from a scatterer which reduces scattering by vapor atoms and ripplons. This reduction can be explained differently considering the moving reference frame, where the cyclotron orbit is at rest. In this reference frame, electron scattering becomes inelastic because of the Doppler correction to the energy exchange at a collision $\hbar \mathbf{q u} f \sim e E_{f} l_{B}$ (here $\mathbf{u}_{f}$ is the drift velocity and $l_{B}$ is the magnetic length, $\left.l_{B}^{2}=\hbar c / e B\right)$. Then, intra-level scattering 
is suppressed, when the energy exchange $\left(\approx e E_{f} l_{B}\right)$ exceeds the collision broadening of Landau levels. In the many-electron transport theory [8,9], both the collision broadening of Landau levels and the quasi-uniform fluctuational electric field were incorporated using the dynamic structure factor of the 2D Coulomb liquid.

Strong suppression of SE scattering induced by internal forces was observed in magnetoconductivity measurements for electron interaction with vapor atoms [10,11], and ripplons [12]. The decrease of the CR linewidth caused by Coulomb interaction is accompanied by a transformation of the CR line shape (from the initial Gaussian to a Lorentzian) in accordance with the many-electron theory [13].

The typical fluctuational electric field $E_{f}^{(0)} \simeq 3 \sqrt{T_{e}} n_{s}^{3 / 4}$ (here $T_{e}$ is the effective electron temperature, and $n_{s}$ is the SE density) increases, when the $2 \mathrm{D}$ electron system is heated by the CR. This means that in a certain range of $n_{s}$, where the many-electron effect is important, major relaxation rates of SEs could be decreasing functions of $T_{e}$. For such a behavior of relaxation rates, the energy balance of the electron system comes into question. Still, by now, heating of strongly interacting SEs by the quantum CR was not theoretically investigated.

In this work, we report results of theoretical study of the energy balance equation of SEs on liquid helium in the quantum regime of CR excitation, when the CR linewidth and the energy relaxation rate are affected by the manyelectron effect. The results obtained here indicate that strong Coulomb interaction, increasing with $n_{s}$, can induce temperature bistability of SEs populating the ground surface subband, if the amplitude of the microwave (MW) field $E_{\mathrm{mw}}^{(0)}$ is strong enough. We found also that in the bistability regime, even a small increase in the MW power can drastically change the line shape $T_{e}(B)$ which should affect the dc magnetoconductivity of SEs.

\section{Basic relations}

\subsection{CR line shape}

Consider the 2D electron system on liquid helium at low temperatures ( $T \sim 0.2 \mathrm{~K})$. The magnetic field directed normally to the surface is assumed to be high $(B>1 \mathrm{~T})$, so that, in the absence of MW irradiation, nearly all electrons occupy the ground Landau level with $n=0$ (the Landau spectrum is defined as $\varepsilon_{n}=\hbar \omega_{c}(n+1 / 2)$, with $n=0,1, \ldots)$. In this regime, the linewidth of the CR is determined by electron interaction with ripplons whose interaction Hamiltonian has the form similar to that of usual electron-phonon interaction

$$
V_{r}=\sum_{\mathbf{q}} V_{r, q} Q_{q}\left(b_{-\mathbf{q}}+b_{\mathbf{q}}^{\dagger}\right) \mathrm{e}^{-i \mathbf{q r}},
$$

where $b_{\mathbf{q}}^{\dagger}$ and $b_{\mathbf{q}}$ are creation and destruction operators of ripplons, $V_{r, q}$ is the electron-ripplon coupling, $Q_{q}^{2}=\hbar q / 2 \rho \omega_{r, q}$, the surface areal is set to unity, and $\omega_{r, q}$ is the ripplon dispersion. For wave-vectors $q<3 \cdot 10^{7} \mathrm{~cm}^{-1}$, the $\omega_{r, q} \simeq \sqrt{\alpha / \rho} q^{3 / 2}$, where $\alpha$ and $\rho$ are the surface tension and mass density of liquid helium, respectively. Under a high magnetic field, momentum conservation usually restricts $q \lesssim l_{B}^{-1}$.

The average MW absorption is proportional to the real part of the electron conductivity $\operatorname{Re} \sigma_{x x}(\omega)$. In the vicinity of the resonance, the memory function method yields [9]

$$
\operatorname{Re} \sigma_{x x}(\omega) \simeq \frac{e^{2} n_{s}}{2 m_{e}} \frac{v_{\mathrm{eff}}(\omega)}{\left(\omega-\omega_{c}\right)^{2}+v_{\mathrm{eff}}^{2}(\omega)},
$$

where

$v_{\text {eff }}(\omega)=\frac{1-\mathrm{e}^{-\hbar \omega / T} e}{4 \hbar \omega m_{e}} \sum_{\mathbf{q}} q^{2} V_{r, q}^{2} Q_{q}^{2}\left(2 N_{q}^{(r)}+1\right) S(q, \omega)$,

$N_{q}^{(r)}$ is the distribution function of ripplons, and $S(q, \omega)$ is the dynamic structure factor (DSF) of the electron liquid. Because electron-electron collisions are quite frequent for SEs on liquid helium, we have used the electron temperature $T_{e}$ approximation to describe electron distribution. In the limiting case $\omega \rightarrow 0$, Eq. (3) describes also the effective collision frequency of the dc conductivity $\sigma_{x x}(0)$ which has a strong dependence on the electron temperature $\sigma_{x x}(0) \propto 1 / T_{e}$. In this case, the correct form of $\sigma_{x x}(0)$ requires the inclusion of the nonresonant term whose denominator has $\omega+\omega_{c}$ instead of $\omega-\omega_{c}$. In Eq. (2), this term was neglected.

For nondegenerate electrons, the single-electron theory yields

$$
\begin{aligned}
& S(q, \Omega)=\frac{2}{\pi \hbar Z_{\|}} \sum_{n, n^{\prime}} I_{n, n^{\prime}}^{2}\left(x_{q}\right) \times \\
& \times \int \mathrm{e}^{-\varepsilon / T_{e}} g_{n}(\varepsilon) g_{n^{\prime}}(\varepsilon+\hbar \Omega) d \varepsilon,
\end{aligned}
$$

where $g_{n}(\varepsilon)=-\operatorname{Im} G_{n}(\varepsilon)$ represents the Landau level density of states, $G_{n}(\varepsilon)$ is the single-electron Green's function, $x_{q}=q^{2} l_{B}^{2} / 2$,

$$
I_{n, n^{\prime}}^{2}\left(x_{q}\right)=\frac{\min \left(n, n^{\prime}\right) !}{\max \left(n, n^{\prime}\right) !} x_{q}^{\left|n^{\prime}-n\right|} \mathrm{e}^{-x_{q}}\left[L_{\min \left(n, n^{\prime}\right)}^{\left|n^{\prime}-n\right|}\left(x_{q}\right)\right]^{2} \text {, }
$$

and $L_{n}^{m}(x)$ are the associated Laguerre polynomials. The cumulant expansion method [14] gives the Gaussian shape

$$
g_{n}(\varepsilon)=\frac{\sqrt{2 \pi} \hbar}{\Gamma_{n}} \exp \left[-\frac{2\left(\varepsilon_{n}-\varepsilon\right)^{2}}{\Gamma_{n}^{2}}\right]
$$

which is very useful for obtaining the analytical form of the DSF. 
For SEs with strong Coulomb interaction, the correct form of the DSF was found considering an ensemble of electrons with orbit centers moving fast due to the quasiuniform fluctuational electric field [9]

$$
S(q, \Omega)=\frac{2 \sqrt{\pi}}{Z_{\|}} \sum_{n, n^{\prime}} \frac{I_{n, n^{\prime}}^{2}\left(x_{q}\right)}{\gamma_{n, n^{\prime}}} \exp \left[-\frac{\varepsilon_{n}}{T_{e}}-P_{n, n^{\prime}}(\Omega)\right],
$$

where

$$
\begin{gathered}
P_{n, n^{\prime}}(\Omega)=\frac{\left[\Omega-\left(n^{\prime}-n\right) \omega_{C}-\varphi_{n}\right]^{2}}{\gamma_{n, n^{\prime}}^{2}} ; \\
\varphi_{n}=\frac{\Gamma_{n}^{2}+x_{q} \Gamma_{C}^{2}}{4 T_{e} \hbar} ; \\
\gamma_{n, n^{\prime}}=\sqrt{\frac{\Gamma_{n}^{2}+\Gamma_{n^{\prime}}^{2}}{2 \hbar^{2}}+x_{q} \frac{\Gamma_{C}^{2}}{\hbar^{2}}},
\end{gathered}
$$

and $\Gamma_{C}=\sqrt{2} e E_{f}^{(0)} l_{B}$. As compared to the single-electron treatment, the fluctuational electric field induces an additional broadening of the DSF of the electron liquid which depends on $q, n_{s}$, and electron temperature: $\sqrt{x_{q}} \Gamma_{C} / \hbar$.

There is also an additional frequency shift $x_{q} \Gamma_{C}^{2} / 4 T_{e} \hbar$ which is independent of $T_{e}$.

The Eqs. (2)-(9) describe well the transformation of the CR line shape of SEs caused by internal forces in different experiments $[2,3,13]$. We shall use these equations to obtain the temperature dependence of MW absorption. Under magnetic field, it is convenient to introduce the dimensionless coupling function [15]

$$
V_{1,1}\left(x_{q}\right) \equiv \frac{l_{B}^{2}}{\Lambda} V_{r, q}=x_{q} u_{C}\left(\frac{x_{q}}{2 \beta^{2} l_{B}^{2}}\right)+\frac{e E_{\perp} l_{B}^{2}}{\Lambda},
$$

where $\Lambda=e^{2}(\epsilon-1) / 4(\epsilon+1), \epsilon$ is the dielectric constant of liquid helium, $\beta$ is the parameter of the $\mathrm{SE}$ wave function $f_{1}(z)=2 \beta^{3 / 2} z \exp (-\beta z)$, and

$$
u_{c}(x)=-\frac{1}{1-x}+\frac{1}{(1-x)^{3 / 2}} \ln \left(\frac{1+\sqrt{1-x}}{\sqrt{x}}\right) .
$$

In the following we shall consider the regime $\Gamma_{C}<<\hbar \omega_{C}$. Then, neglecting exponentially small terms, one can obtain

$$
\begin{gathered}
v_{\text {eff }}(\omega)=\frac{\left(1-\mathrm{e}^{-\hbar \omega / T_{e}}\right) T \Lambda^{2}}{4 \sqrt{\pi} \hbar \omega \alpha m_{e} l_{B}^{6}} \times \\
\times \sum_{n=0}^{\infty} \frac{\mathrm{e}^{-\varepsilon_{n} / T_{e}}}{(n+1) Z_{\|}} \int_{0}^{\infty} d x_{q} V_{1,1}^{2}\left(x_{q}\right) \frac{x_{q}}{\gamma_{n, n+1}} \times \\
\times \mathrm{e}^{-x_{q}}\left[L_{n}^{1}\left(x_{q}\right)\right]^{2} \exp \left[-\frac{\left(\omega-\omega_{c}-\varphi_{n}\right)^{2}}{\gamma_{n, n+1}^{2}}\right],
\end{gathered}
$$

where $Z_{\|}$is the partition function.
The dependence $v_{\text {eff }}(\omega) \propto 1 / \gamma_{n, n+1}$ reflects the singular nature of the quantum magnetotransport in $2 \mathrm{D}$ systems. In the single-electron theory, it yields the enhancement factor $\approx \hbar \omega_{c} / \Gamma_{n}$. The Coulomb broadening, entering the definition of $\gamma_{n, n+1}$ in Eq. (9), describes the reduction of electron scattering caused by the fluctuational electric field. Thus, Eq. (12) indicates that at the resonance $v_{\text {eff }}\left(\omega_{c}\right)$ decreases with $T_{e}$ because of the Coulomb broadening in $\gamma_{n, n+1}$, while $\operatorname{Re} \sigma_{x x}\left(\omega_{c}\right)$ and MW absorption increase with $T_{e}$.

\subsection{Energy relaxation rates}

The amount of energy taken by SEs from the MW field should be balanced by the amount transferred to ripplons. The average probability of electron scattering with the momentum exchange $\hbar \mathbf{q}$ caused by one-ripplon creation $(+)$ and destruction (-) can be also expressed in terms of the DSF [16]

$$
v_{\mathbf{q}}^{( \pm)}=\frac{V_{r, q}^{2} Q_{q}^{2}}{\hbar^{2}}\left(N_{q}^{(r)}+1 / 2 \pm 1 / 2\right) S\left(q,-q_{y} V_{H} \mp \omega_{r, q}\right),
$$

where $V_{H}$ is the Hall velocity in the external dc electric field. This representation is similar to that of the theory of thermal neutron (or x-ray) scattering by solids, where the scattering cross section of a particle flux is expressed in terms of the DSF of the target. The many-electron form of the DSF given in Eq. (7) follows also from Eq. (13), if we use averaging over the drift velocities in the fluctuational electric field [8,9]. For energy relaxation, we can set $V_{H} \rightarrow 0$, assuming that $S(q, \Omega)$ is the DSF of interacting electrons.

Using quantities $v_{\mathbf{q}}^{( \pm)}$, the amount of energy transferred to ripplons due to one-ripplon scattering by an electron per unit time can be defined as

$$
\dot{w}_{1 r}=\sum_{\mathbf{q}} \hbar \omega_{r, q}\left[v_{\mathbf{q}}^{(+)}-v_{\mathbf{q}}^{(-)}\right] .
$$

Equilibrium properties of $S(q, \Omega)$ allow us to obtain

$$
\begin{aligned}
\dot{w}_{1 r}= & \frac{1}{\hbar^{2}} \sum_{\mathbf{q}} \hbar \omega_{r, q} V_{r, q}^{2} Q_{q}^{2} N_{q}^{(r)} S\left(q, \omega_{r, q}\right) \times \\
\times & {\left[\mathrm{e}^{\hbar \omega_{r, q} / T-\hbar \omega_{r, q} / T_{e}}-1\right] . }
\end{aligned}
$$

The energy exchange $\hbar \omega_{r, q}$ is usually much lower than $T$. Therefore, $\dot{w}_{1 r}$ can be represented as $\dot{w}_{1 r}=\left(T_{e}-T\right) \tilde{v}_{1 r}$, where $\tilde{v}_{1 r}$ is the energy relaxation rate. Neglecting exponentially small terms (we consider $\Gamma_{C}<<\hbar \omega_{C}$ ), $\tilde{v}_{1 r}$ can be found as

$$
\begin{gathered}
\tilde{v}_{1 r}=\frac{\Lambda^{2}}{\sqrt{2 \pi} \rho l_{B}^{7} T_{e}} \frac{1}{Z_{\|}} \sum_{n=0}^{\infty} \mathrm{e}^{-\varepsilon_{n} / T_{e}} \times \\
\times \int_{0}^{\infty} d x_{q} x_{q}^{1 / 2} V_{1,1}^{2}\left(x_{q}\right) \mathrm{e}^{-x_{q}} \frac{\left[L_{n}\left(x_{q}\right)\right]^{2}}{\gamma_{n, n}} .
\end{gathered}
$$


One can see that $\left(T_{e}-T\right) \tilde{v}_{1 r}$ eventually becomes a decreasing function of $T_{e}$ because of the Coulomb contribution into $\gamma_{n, n}$ and the singular nature of one-ripplon scattering of SEs under a high magnetic field.

In one-ripplon scattering processes, the energy exchange $\hbar \omega_{r, q}$ is small because the ripplon wave vector is restricted by the inverse magnetic length: $q \lesssim l_{B}^{-1}$. High energy ripplons can take part in two-ripplon scattering events, when an electron creates a couple of short wavelength ripplons [17-19] with the total momentum $\mathbf{s}=\mathbf{q}+\mathbf{q}^{\prime}$ restricted by the condition $s \lesssim l_{B}^{-1}<<q$. This can lead to a much higher energy relaxation rate. Recently, this theoretical prediction was confirmed experimentally [20].

In the presence of a high magnetic field, the energy exchange $\left(\simeq 2 \hbar \omega_{r, q}\right)$ of a couple of short wavelength ripplons is much larger than the Landau level broadening $\Gamma_{n}$, and, therefore, such two-ripplon processes can not take place for electron scattering within the Landau level. They can substantially contribute to energy relaxation, when the system is heated, and electron scattering between different Landau levels becomes important $\left(T_{e} \gtrsim \hbar \omega_{c}\right)$.

Two-ripplon scattering processes noted above are described by the following Hamiltonian

$$
\begin{gathered}
H_{2 r}=\sum_{\mathbf{q}, \mathbf{q}^{\prime}} W_{q} Q_{q} Q_{q^{\prime}}\left(b_{-\mathbf{q}}+b_{\mathbf{q}}^{\dagger}\right) \times \\
\times\left(b_{-\mathbf{q}^{\prime}}+b_{\mathbf{q}^{\prime}}^{\dagger}\right) \mathrm{e}^{-i\left(\mathbf{q}+\mathbf{q}^{\prime}\right) \mathbf{r}} .
\end{gathered}
$$

The coupling function $W_{q}$ is usually interpolated employing the unit step-function [19]

$$
W_{q} \simeq W_{q}^{(\mathrm{lon})} \theta\left(q^{*}-q\right)+W_{q}^{(\mathrm{sh})} \theta\left(q-q^{*}\right)
$$

joining two asymptotes

$$
W_{q}^{(\text {lon })} \simeq\left(\frac{\hat{p}_{z}^{2}}{2 m_{e}}\right)_{1,1} q^{2}, \quad W_{q}^{(\mathrm{sh})}=V_{0} \varkappa^{-1}\left[f_{1}^{\prime}(0)\right]^{2},
$$

where $q^{*} \simeq 1.65 \cdot 10^{7} \mathrm{~cm}^{-1}, V_{0}$ is the repulsion barrier at the interface, $\varkappa^{-1}=\hbar / \sqrt{2 m_{e} V_{0}} \simeq 2 \cdot 10^{-8} \mathrm{~cm}$ is the penetration length of the SE wave function $f_{1}(z)$ into the liquid phase, $f_{1}^{\prime}(0)$ is the derivative at the interface, $\hat{p}_{z}$ is the electron momentum in the perpendicular direction, and $(\ldots)_{1,1}$ means averaging over the ground surface-state wave function $f_{1}(z)$. Since $s \lesssim l_{B}^{-1}<<q$, the absolute value $q^{\prime} \simeq q$. We shall consider mostly high magnetic fields, when the wavevector defined by energy conservation $\left[2 \omega_{r, q}=\left(n-n^{\prime}\right) \omega_{c}\right]$ is larger than $q^{*}$.

For the interaction Hamiltonian of Eq. (16), the average amount of energy lost by an electron per unit time due to two-ripplon scattering can be found using the Born approximation

$$
\begin{gathered}
\dot{w}_{2 r}=\frac{4 \pi}{\hbar} \sum_{\mathbf{q}, \mathbf{s}} 2 \hbar \omega_{r, q} W_{q}^{2} Q_{q}^{4} \times \\
\times \sum_{n, n^{\prime}} \frac{\mathrm{e}^{-\varepsilon_{n} / T} e}{Z_{\|}} I_{n, n^{\prime}}^{2}\left(x_{s}\right)\left(N_{q}+1\right)^{2} \times \\
\times\left[\delta\left(\varepsilon_{n^{\prime}}-\varepsilon_{n}+2 \hbar \omega_{r, q}\right)-\mathrm{e}^{-2 \hbar \omega_{r, q} / T} \delta\left(\varepsilon_{n}-\varepsilon_{n}-2 \hbar \omega_{r, q}\right)\right] .
\end{gathered}
$$

Under the condition $s<<q$ used above, summation over s can be done analytically $\sum_{\mathbf{s}} I_{n, n^{\prime}}^{2}\left(x_{s}\right)=1 / 2 \pi l_{B}^{2}$ (the sur-

face areal is set to unity), and Eq. (19) can be reduced to simple sums

$$
\begin{gathered}
\dot{w}_{2 r}=\frac{\hbar}{4 \pi \rho^{2} l_{B}^{2}} \sum_{n=1}^{\infty} \sum_{k=1}^{n} \frac{\mathrm{e}^{-\varepsilon_{n} / T_{e}}}{Z_{\|}} \times \\
\times\left\{\frac{q^{3} W_{q}^{2}}{\omega_{r, q}\left|\omega_{r, q}^{\prime}\right|}\left(N_{q}+1\right)^{2}\left[1-\mathrm{e}^{-2 \hbar \omega_{r, q}\left(1 / T-1 / T_{e}\right)}\right]\right\}_{q=q_{k}},
\end{gathered}
$$

where $q_{k}$ is the solution of the equation $2 \omega_{r, q}=k \omega_{c}$ (here $k=n-n^{\prime}$ ). The Eq. (20) indicates that two-ripplon scattering under magnetic field is not singular: a finite result is found in the limiting case $\Gamma_{n} \rightarrow 0$. This means that the influence of the fluctuational electric field on tworipplon scattering can be neglected if $\Gamma_{C}<<\hbar \omega_{c}$.

The result of Eq. (20) depends strongly on the ripplon dispersion in the short wavelength range. Experimental data [21] and theoretical analysis [22-24] indicate that the ripplon dispersion curve should remain below the roton threshold $\Delta \simeq 8.6 \mathrm{~K}$ because of interaction with bulk rotons. It is obvious that in this case, $\omega_{q}^{\prime}$ decreases fast at large $q$ which should increase the energy loss of Eq. (20). In our calculations, we shall use mostly two extreme models of ripplon dispersion at large wave vectors: 1$)$ the pure capillary dispersion with $\omega_{r}(q)=\sqrt{\alpha / \rho} q^{3 / 2} ; 2$ ) the simple analytical interpolation

$$
\tilde{\omega}_{r}(q)=\frac{\omega_{\Delta} \omega_{r}(q)}{\sqrt{\omega_{r}^{2}(q)+\omega_{\Delta}^{2}}}, \quad \omega_{\Delta}=\frac{\Delta}{\hbar}
$$

which is quite close to experimental data [21]. For the second model, the solution $q_{k}$ is found as

$$
q_{k}=\left(\frac{\rho}{4 \alpha}\right)^{1 / 3} \frac{\left(k \omega_{c}\right)^{2 / 3}}{\left(1-\left(k \omega_{c} / 2 \omega_{\Delta}\right)^{2}\right)^{1 / 3}} \theta\left(1-\frac{k \omega_{c}}{2 \omega_{\Delta}}\right) .
$$

In the limit $k \omega_{c} / 2 \omega_{\Delta}<<1$, Eq. (22) transforms into the solution $q_{k}=(\rho / 4 \alpha)^{1 / 3}\left(k \omega_{c}\right)^{2 / 3}$ valid for the pure ripplon dispersion (the first model). Under the condition $2 \omega_{\Delta} / \omega_{c} \rightarrow k$, the $q_{k} \rightarrow \infty$. In this case, we shall restrict $q$ by the posi- 
tion of the roton minimum: $q \leq q_{0} \simeq 1.9 \cdot 10^{8} \mathrm{~cm}^{-1}$. Alternatively, one could assume that at $q>q_{0}$ the ripplon dispersion curve follows (slightly below) the roton dispersion curve. The $\dot{w}_{2 r}(B)$ calculated for these two alternatives is shown in Fig. 1 by solid $\left.\left(q_{k} \leq q_{0}\right)\right)$ and dotted ( $q_{k}$ can be larger than $q_{0}$ ) curves, respectively. Dashed curves represent $\dot{w}_{2 r}$ for the pure capillary dispersion (the first model). Thus, at high $T_{e}$ the second dispersion model results in sharp maxima of $\dot{w}_{2 r}$ whose positions are defined by the condition $2 \tilde{\omega}_{r}\left(q_{0}\right) / \omega_{c} \rightarrow$ integer.

Different contributions to the average energy transferred to ripplons by an electron per unit time are shown in Fig. 2, as the functions of the electron temperature. In the singleelectron treatment (S-E), the contribution from one-ripplon scattering $\dot{w}_{1 r}$ increases strongly with $T_{e}$. In the manyelectron theory (M-E), the same quantity becomes a decreasing function at $T_{e}>0.7 \mathrm{~K}$. The two-ripplon contribution $\dot{w}_{2 r}$ is very small at low temperatures $T_{e}<1.5 \mathrm{~K}$ because excited Landau levels are nearly empty. At high temperatures $T_{e}>1.5 \mathrm{~K}, \quad \dot{w}_{2 r}$ dominates and the total energy loss of SEs becomes an increasing function of $T_{e}$.

The energy gain of SEs from the MW field should be balanced by the energy loss caused by one-ripplon and two-ripplon scattering processes. This yields the energy balance equation

$$
\frac{e^{2}}{4 m_{e}} \frac{v_{\mathrm{eff}}(\omega)\left(E_{\mathrm{mw}}^{(0)}\right)^{2}}{\left(\omega-\omega_{c}\right)^{2}+v_{\mathrm{eff}}^{2}(\omega)}=\left(T_{e}-T\right) \tilde{v}_{1 \mathrm{r}}+\dot{w}_{2 \mathrm{r}},
$$

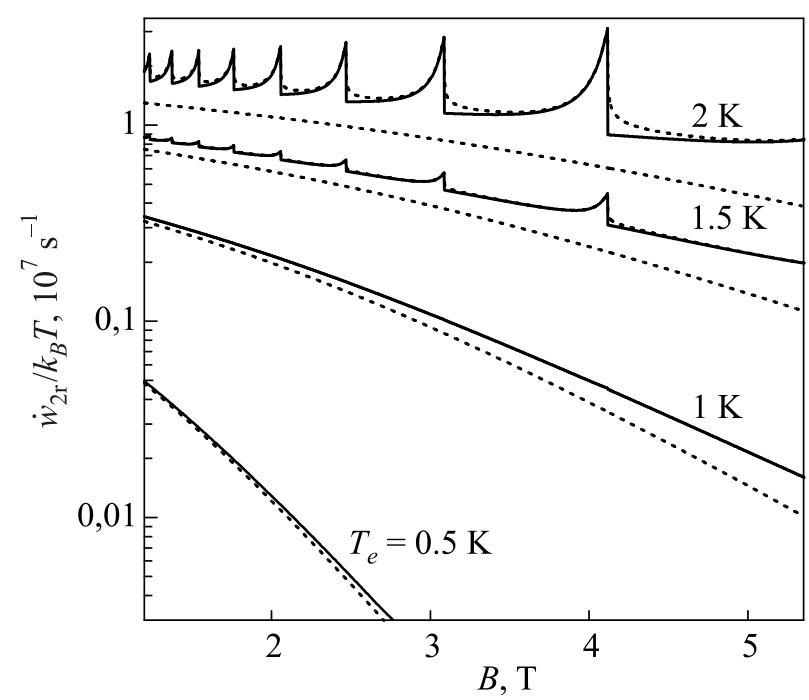

Fig. 1. $\dot{w}_{2 r} / k_{B} T$ vs $B$ calculated for $T=0.2 \mathrm{~K}$ and four electron temperatures: $T_{e}=0.5,1.0,1.5$, and $2 \mathrm{~K}$. Dashed lines are obtained using the pure capillary dispersion, solid lines are for the model dispersion $\tilde{\omega}_{r}(q)$ of Eq. (21) ending at $q=q_{0}$, dotted lines are calculated for the model $\tilde{\omega}_{r}(q)$ which follows the roton dispersion at $q>q_{0}$, as described in the text.

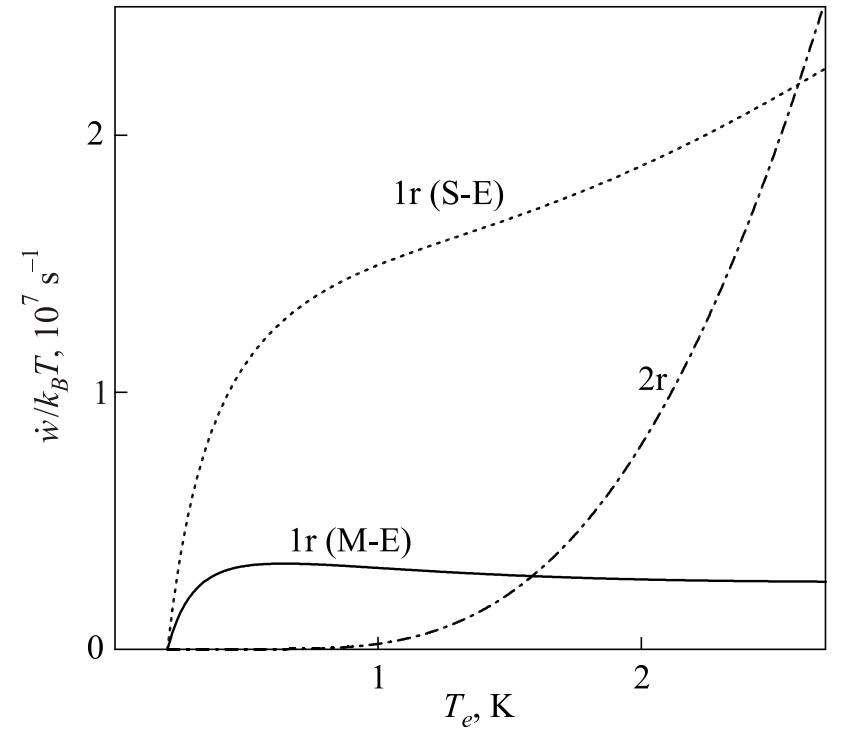

Fig. 2. Different contributions into $\dot{w}$ vs $T_{e}$ calculated for $T=0.2 \mathrm{~K}, B=5 \mathrm{~T}$, and $n_{S}=50 \cdot 10^{6} \mathrm{~cm}^{-2}$ : one-ripplon scattering of the single-electron theory (dashed), one-ripplon scattering of the many-electron theory (solid), two-ripplon scattering for the ripplon dispersion of Eq. (21) (dash-dotted).

where $E_{\mathrm{mw}}^{(0)}$ is the amplitude of the MW field. This equation defines the electron temperature as the function of $E_{\mathrm{mw}}^{(0)}$ and $B$. Since $\dot{w}_{2 r}$ becomes important only when $T_{e} \gtrsim \hbar \omega_{c}$, to reach temperature bistability one have to use a high enough magnetic field.

\section{Results and discussion}

The dependence $T_{e}\left(E_{\mathrm{mw}}^{(0)}\right)$ defined by the energy balance equation can be found trivially by inverting the obvious relationship $E_{\mathrm{mw}}^{(0)}\left(T_{e}\right)$ which directly follows from Eq. (23). Under the CR condition $\left(\omega_{c}=\omega\right)$ )which occurs at a high magnetic field $(B=5 \mathrm{~T})$, the dependence $T_{e}\left(E_{\mathrm{mw}}^{(0)}\right)$ is shown in Fig. 3. The results obtained for the ripplon dispersion model 1 and the dispersion model 2 are shown by dashed and solid curves, respectively. In the case of the single-electron theory, the curves marked with S-E show no bistability which means that at low SE densities one can use quite strong $\mathrm{MW}$ fields $\left(E_{\mathrm{mw}}^{(0)} \simeq 0.06 \mathrm{~V} / \mathrm{cm}\right)$ without a substantial increase in population of excited surface subbands. For the many-electron theory, the $T_{e}\left(E_{\mathrm{mw}}^{(0)}\right)$ curves depend strongly on electron density. For two values of $n_{s}$ chosen in Fig. 3, the many electron curves demonstrate temperature bistability. The bistability range increases with electron density. In is important that both the lowtemperature and high-temperature branches of the bistability regime are below $2 \mathrm{~K}$ which means that nearly all SEs occupy the ground surface subband. The difference between two ripplon dispersion models discussed above becomes important only for the high temperature branch.

At a lower magnetic field $(B=2 \mathrm{~T})$, the evolution of the $T_{e}\left(E_{\mathrm{mw}}^{(0)}\right)$ curve with an increase in electron density is 


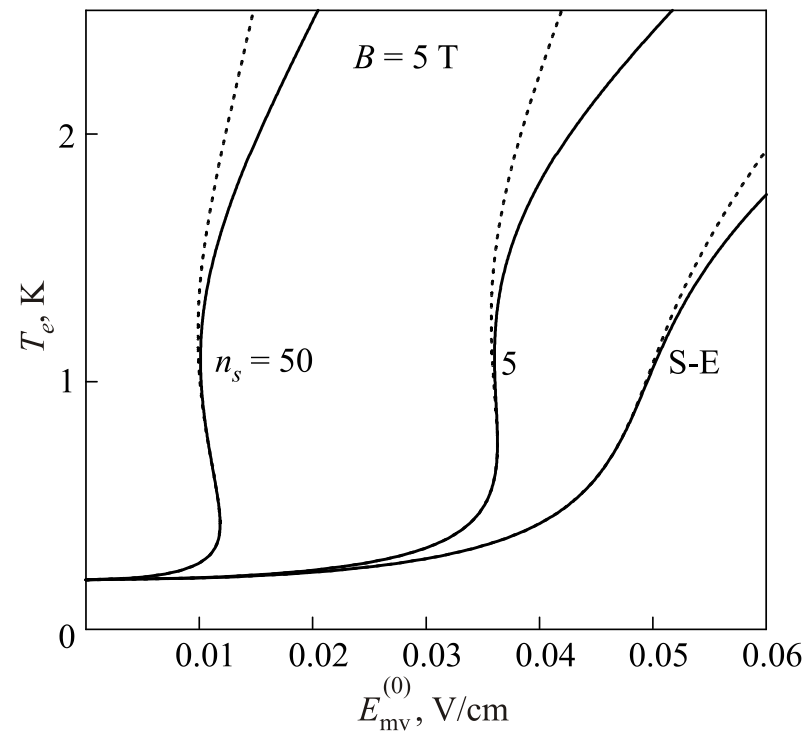

Fig. 3. $T_{e}$ vs $E_{\mathrm{mw}}^{(0)}$ calculated for $T=0.2 \mathrm{~K}, B=5 \mathrm{~T}$, and different electron densities: the single-electron theory (S-E), and the many-electron theory for $n_{s}=5 \cdot 10^{6}$ and $50 \cdot 10^{6} \mathrm{~cm}^{-2}$. The dashed lines are calculated for the pure capillary spectrum. The solid lines are found for the ripplon dispersion model of Eq. (21).

shown in Fig. 4. In this case, bistability vanishes, still the increase of $T_{e}$ with $E_{\mathrm{mw}}^{(0)}$ becomes sharper for higher densities. This explains the fast increase of the electron temperature and evaporation of SEs from the ground surface subband into 3D states observed experimentally at low magnetic fields and high densities [2,3,25].

To obtain the electron temperature as a function of the magnetic field, numerical solution of Eq. (23) is necessary.

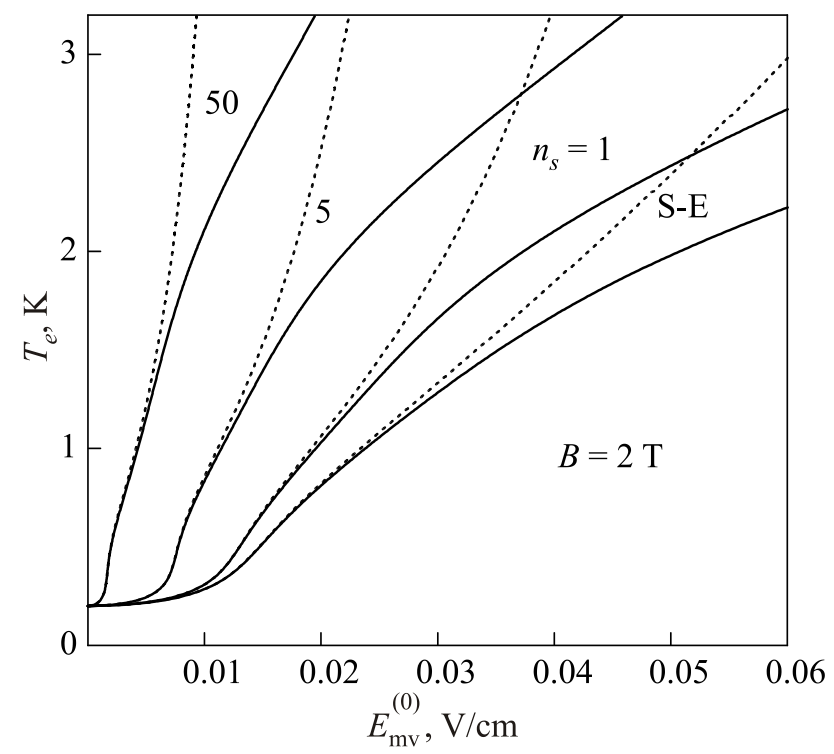

Fig. 4. $T_{e}$ vs $E_{\mathrm{mw}}^{(0)}$ calculated for $T=0.2 \mathrm{~K}, B=2 \mathrm{~T}$, and different electron densities: the single-electron theory (S-E), and the manyelectron theory for $n_{S}=1 \cdot 10^{6}, 5 \cdot 10^{6}$, and $50 \cdot 10^{6} \mathrm{~cm}^{-2}$. The dashed lines are calculated for the pure capillary spectrum. The solid lines are found for the ripplon dispersion model of Eq. (21).
The evolution of the line shape $T_{e}(B)$ with a gradual increase in $E_{\mathrm{mw}}^{(0)}$ for $E_{\mathrm{mw}}^{(0)} \leq E_{\mathrm{th}} \simeq 0.011796 \mathrm{~V} / \mathrm{cm}$ is shown in Fig. 5 (here $E_{\text {th }}$ is the threshold field for the bistability jump to the high-temperature branch). The top of the curve $T_{e}(B)$ becomes sharper, when $E_{\mathrm{mw}}^{(0)}$ approaches $E_{\mathrm{th}}$. The line shape of $T_{e}(B)$ drastically changes when $E_{\mathrm{mw}}^{(0)}$ becomes larger than $E_{\text {th }}$, as shown in Fig. 6. Slightly above the threshold field $\left(E_{\mathrm{mw}}^{(0)}=0.012 \mathrm{~V} / \mathrm{cm}\right)$ the dependence $T_{e}(B)$ has two bistability jumps to the high-temperature branch and two bistability jumps back to the lowtemperature branch. Therefore, the shape of $T_{e}(B)$ depends on the history of past changes of $B$ (the direction of a sweep of $B$ ) and demonstrates a hysteresis. A line shape $T_{e}(B)$, corresponding to monotonic changes of $B$ (upward or downward), is not symmetrical with respect to the position of the CR.

It is interesting that even a small increase in the MW field (from 0.012 to $0.015 \mathrm{~V} / \mathrm{cm}$ ) eliminates temperature bistability of the line shape $T_{e}(B)$, as shown in Fig. 6 . This behavior is cased by the term $\left(\omega-\omega_{c}\right)^{2}$ in the denominator of the left side of Eq. (23). A substantial increase in it starts inverting the dependence of $\operatorname{Re} \sigma_{x x}$ on $v_{\text {eff }}$ which results in a stable solution of the energy balance equation.

The roton threshold of the ripplon dispersion $\omega_{\Delta}$ should affect the dependence $T_{e}(B)$, when the MW frequency is close to $2 \omega_{\Delta} / k$ and $E_{\mathrm{mw}}^{(0)}>E_{\mathrm{th}}$. This conclusion follows from solid and dotted lines of Fig. 1 which have sharp maxima at certain values of the magnetic field.

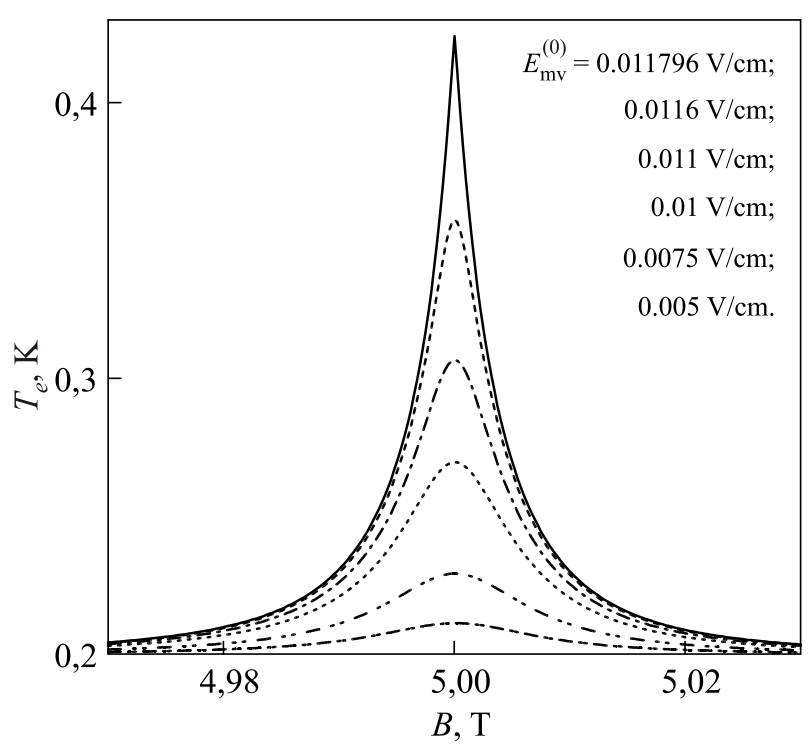

Fig. 5. $T_{e}$ vs $B$ calculated for the ripplon dispersion model of Eq. (21), $T=0.2 \mathrm{~K}, n_{\mathrm{s}}=50 \cdot 10^{6} \mathrm{~cm}^{-2}$, and different $E_{\mathrm{mw}}^{(0)}$ below the threshold field $E_{\mathrm{th}}$. The lowest line corresponds to $E_{\mathrm{mw}}^{(0)}=0.005 \mathrm{~V} / \mathrm{cm}$. 


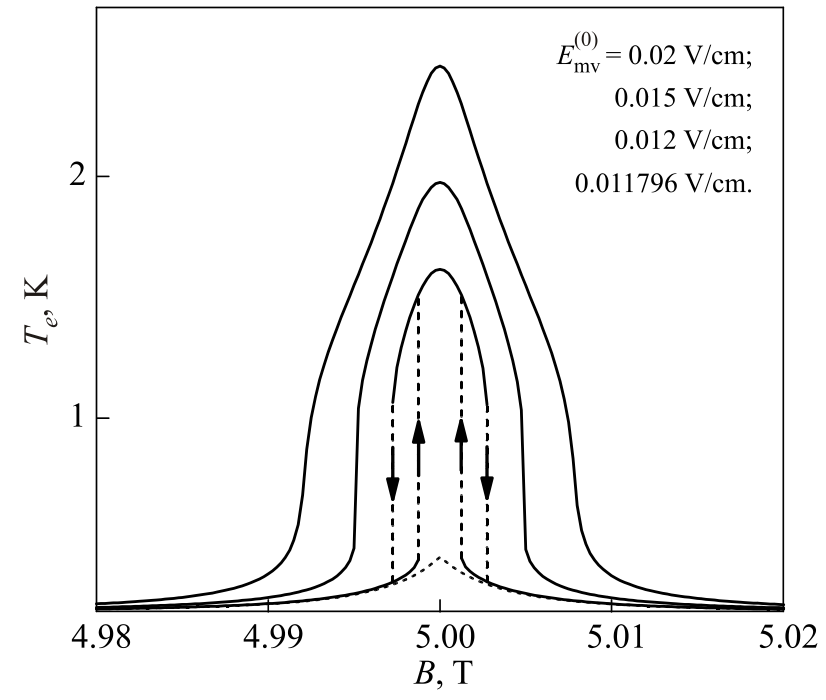

Fig. 6. $T_{e}$ vs $B$ calculated for the ripplon dispersion model of Eq. (21), $T=0.2 \mathrm{~K}, n_{s}=50 \cdot 10^{6} \mathrm{~cm}^{-2}$, and different $E_{\mathrm{mw}}^{(0)}$ above the threshold field. The lowest line (dotted) corresponds to $E_{\mathrm{mw}}^{(0)}=E_{\mathrm{th}}$ (the highest line of Fig. 5).

The final result depends strongly on the ripplon dispersion at $q>q_{0}$. If the ripplon dispersion $\tilde{\omega}_{r}\left(q_{0}\right)$ ends at $q=q_{0}$ or just remains below $\omega_{\Delta}$ at $q>q_{0}$ as well, then the abrupt reduction in $\dot{w}_{2 r}$ at $k \omega_{c}(B)>2 \tilde{\omega}_{r}\left(q_{0}\right)$ or at $k \omega_{C}(B)>2 \omega_{\Delta}$ leads to an additional sharp maximum of the line shape $T_{e}(B)$. This additional maximum is substantially reduced, if we assume that at $q>q_{0}$ the ripplon dispersion follows the roton dispersion $\left(\dot{w}_{2 r}\right.$ changes according to dotted lines of Fig. 1).

The dependence $T_{e}(B)$ obtained here should display itself in dc magnetoconductivity measurements conducted under CR excitation, because $\sigma_{x x}^{(\mathrm{dc})} \propto v_{\text {eff }}(0) / \omega_{c}^{2} \propto 1 / T_{e}$. It is obvious that $\sigma_{X X}^{(\mathrm{dc})}$, as a function of $B$, should also have bistability jumps, when $E_{\mathrm{mw}}^{(0)}$ slightly exceeds the threshold value $E_{\text {th }}$. Recent studies [26] indicate that photonassisted scattering by ripplons results in a sign-changing correction to $\sigma_{x x}^{(\mathrm{dc})}$ whose strength increases in the vicinity of the CR condition. Therefore, at the minimum of the line $\sigma_{X x}^{(\mathrm{dc})}(B)$ determined by $1 / T_{e}(B)$, the sign-changing correction can induce an additional wavy structure, depending on $n_{s}$ due to the fluctuational electric field. The accurate evaluation of dc conductivity changes under CR excitation caused by photon-assisted scattering of hot electrons requires an additional study, and will be given elsewhere.

\section{Conclusions}

Under CR excitation, heating of SEs on liquid helium is shown to increase fast with electron density due to the Coulombic effect. This conclusion follows from the singular nature of the quantum magnetotransport in 2D systems. The internal electric field of fluctuational origin suppresses energy relaxation of SEs caused by one-ripplon scattering and increases MW absorption at the CR. Therefore, at high magnetic fields, Coulomb interaction induces temperature bistability of electrons, occupying the ground surface subband. The line shape of electron temperature, as a function of the magnetic field, transforms drastically, when the MW field passes over the threshold value. In a narrow range of the MW field amplitude, this line shape acquires a hysteresis with bistability jumps. At high electron temperatures (about $2 \mathrm{~K}$ or higher), the energy relaxation rate of SEs is sensitive to details of the ripplon dispersion at large wave numbers near the roton threshold. This can be used for an alternative experimental investigation of the dispersion of short wavelength ripplons.

1. T.R. Brown and C.C. Grimes, Phys. Rev. Lett. 29, 1233 (1972).

2. V.S. Edel'man, Zh. Eksp. Teor. Fiz. 77, 673 (1979) [Sov. Phys. JETP 50, 338 (1979)].

3. V.S. Edel'man, Usp. Fiz. Nauk 130, 675 (1980) [Sov. Phys. Usp. 23, 227 (1980)].

4. T. Aoki and M. Saitoh, J. Phys. Soc. Jpn. 48, 1929 (1980).

5. T. Ando and Y. Uemura, J. Phys. Soc. Jpn. 36, 959 (1974)

6. M.I. Dykman and L.S. Khazan, Zh. Eksp. Teor. Fiz. 77, 1488 (1979) [Sov. Phys. JETP 50, 747 (1979)].

7. C. Fang-Yen, M.I. Dykman, and M.J. Lea, Phys. Rev. B 55, 16272 (1997).

8. Yu.P. Monarkha, E. Teske, and P. Wyder, Phys. Rep. 370, 161 (2002).

9. Yu.P. Monarkha and K. Kono, Two-Dimensional Coulomb Liquids and Solids, Springer Verlag, Berlin-Heildelberg (2004).

10. M.J. Lea, P. Fozooni, A. Kristensen, P.J. Richardson, K. Djerfi, M.I. Dykman, C. Fang-Yen, and A. Blackburn, Phys. Rev. B 55, 16280 (1997).

11. Yu.P. Monarkha, E. Teske, and P. Wyder, Phys. Rev. B 59, 14884 (1999).

12. Yu.P. Monarkha, S. Ito, K. Shirahama, and K. Kono, Phys. Rev. Lett. 78, 2445 (1997).

13. Yu.P. Monarkha, E. Teske, and P. Wyder, Phys. Rev. B 62, 2593 (2000).

14. R.R. Gerhardts, Surf. Sci. 58, 227 (1976).

15. Yu.P. Monarkha, Fiz. Nizk. Temp. 38, 579 (2012) [Low Temp. Phys. 38, 451 (2012)].

16. Yu.P. Monarkha, Fiz. Nizk. Temp. 39, 1068 (2013) [Low Temp. Phys. 39, 828 (2013)].

17. Yu.P. Monarkha, Fiz. Nizk. Temp. 4, 1093 (1978) [Sov. J. Low Temp. Phys. 4, 515 (1978)].

18. Yu.P. Monarkha and S.S. Sokolov, Fiz. Nizk. Temp. 32, 1278 (2006) [Low Temp. Phys. 32, 970 (2006)].

19. Yu.P. Monarkha, S.S. Sokolov, A.V. Smorodin, and N. Studart, Fiz. Nizk. Temp. 36, 711 (2010) [Low Temp. Phys. 36, 565 (2010)].

20. A.O. Badrutdinov, D. Konstantinov, M. Watanabe, and K. Kono, Europhys. Lett. 104, 47007 (2013). 
21. H.J. Lauter, H. Godfrin, V.L.P. Frank, and P. Leiderer, Phys. Rev. Lett. 68, 2484 (1992).

22. L. Pitaevskii and S. Stringari, Phys. Rev. B 45, 13133-5 (1992).

23. K.A. Gernoth and M.L. Ristig, Phys. Rev. B 45, 2969 (1992).
24. I.N. Adamenko, K.E. Nemchenko, and I.V. Tanatarov, J. Phys.: Conf. Ser. 150, 032107 (2009).

25. A.O. Badrutdinov, L.V. Abdurakhimov, and D. Konstantinov, Phys. Rev. B 90, 075305 (2014).

26. Yu.P. Monarkha, Phys. Rev. B 91, 121402(R) (2015). 